\title{
DISEÑO Y CONSTRUCCIÓN DE UNA FUENTE DE ALTA TENSIÓN PARA LA EXCITACIÓN TRANSVERSAL Y LONGITUDINAL DE LÁSERES GASEOSOS
}

\section{Omar Ormachea Muñoz y Wendy Morrison Vila}

\section{RESUMEN}

En el presente trabajo de investigación se realizó el diseño y construcción de una fuente de alta tensión para la excitación de emisión coherente de alta intensidad en el diapasón UV en medios gaseosos. El transformador principal fue diseñado con los siguientes parámetros $\mathrm{P}=660 \mathrm{~W}$, voltaje en el primario $\mathrm{U}=220 \mathrm{~V}$ y en el secundario $\mathrm{U}=2200 \mathrm{~V}$, se utiliza un quintuplicador de tensión que consta de 5 condensadores de capacitancias: $\mathrm{C}_{1}=0.05 \mu \mathrm{F} \pm 10 \%$ - $3 \mathrm{kV}, \mathrm{C}_{2}=0.5 \mu \mathrm{F} \pm 10 \%-4 \mathrm{kV}, \mathrm{C}_{3}=0.5 \mu \mathrm{F} \pm 10 \%-4 \mathrm{kV}, \mathrm{C}_{4}=0.25 \mu \mathrm{F} \pm 10 \%-4 \mathrm{kV}$ y $\mathrm{C}_{5}=1 \mu \mathrm{F} \pm 10 \%-4 \mathrm{kV}$. La fuente provee un voltaje máximo de $15 \mathrm{kV}$. Además, se construyó un autotransformador que permite la regulación de voltaje a la entrada de la fuente de alta tensión. Inicialmente, esta fuente será utilizada para la generación de radiación láser en $\mathrm{N}_{2}$ en dos configuraciones: Transversal (TEA) y Longitudinal (TE) a una longitud de onda de $337,1 \mathrm{~nm}$.

Palabras Clave: Opto-Electrónica, Fuente de Alta Tensión, Láseres. 